\title{
Theoretically Motivated Search and Detection of Non-thermal Pulsations from PSRs J1747-2958, J2021+3651, and J1826-1256
}

\author{
Jian $\mathrm{Li}^{1}$ (1), Diego F. Torres ${ }^{2,3,4}$ (1), Francesco Coti Zelati ${ }^{2,3}$, Alessandro Papitto ${ }^{5}$ (i), Matthew Kerr ${ }^{6}$ (1), and Nanda Rea ${ }^{2,3}$ (1) \\ ${ }^{1}$ Deutsches Elektronen Synchrotron DESY, D-15738 Zeuthen, Germany; jian.li@ desy.de \\ ${ }^{2}$ Institute of Space Sciences (ICE, CSIC), Campus UAB, Carrer de Can Magrans, E-08193, Barcelona, Spain \\ Institut d'Estudis Espacials de Catalunya (IEEC), E-08034 Barcelona, Spain \\ ${ }_{5}^{4}$ Institució Catalana de Recerca i Estudis Avançats (ICREA), E-08010, Barcelona, Spain; dtorres@ice.csic.es \\ ${ }^{5}$ INAF-Osservatorio Astronomico di Roma, via di Frascati 33, I-00040 Monte Porzio Catone, Roma, Italy \\ ${ }^{6}$ Space Science Division, Naval Research Laboratory, Washington, DC 20375, USA \\ Received 2018 July 24; revised 2018 October 11; accepted 2018 October 16; published 2018 November 21
}

\begin{abstract}
Based on a theoretical selection of pulsars as candidates for detection at X-ray energies, we present an analysis of archival X-ray observations performed with Chandra and XMM-Newton of PSR J1747-2958 (the pulsar in the "Mouse" nebula), PSR J2021+3651 (the pulsar in the "Dragonfly" nebula), and PSR J1826-1256. X-ray pulsations from PSR J1747-2958 and PSR J1826-1256 are detected for the first time, and a previously reported hint of an X-ray pulsation from PSR J2021+3651 is confirmed with a higher significance. We analyze these pulsars' spectra in regard to the theoretically predicted energy distribution, finding a remarkable agreement, and provide here a refined calculation of the model parameters taking into account the newly derived X-ray spectral data.
\end{abstract}

Key words: X-rays: stars - pulsars: individual (PSR J1747-2958, PSR J2021+3651, PSR J1826-1256)

\section{Introduction}

In a recent article we introduced a model for the high-energy emission of pulsars (Torres 2018). According to this model, pulsed spectra detected in gamma-rays and/or X-rays is produced via synchro-curvature radiation, and can be described using just three physical parameters (accelerating electric field, contrast, and magnetic gradient). Interestingly, it was shown that if the model parameters were adjusted to describe the gamma-ray data, the resulting spectral energy distribution (SED) at lower X-ray energies is a relatively good representation of the spectra therein. In particular, in all cases analyzed by Torres (2018) for which both X-ray and gamma-ray data were available, a fit only to the gamma-ray part of the spectra would miss the measured X-ray flux level by less than a factor of $\sim 2$. This was the case even when one of the parameters - on which we comment below-was fixed to an average value (gammaray data alone is not constraining enough to fix it), and thus the spectral shape is completely determined by two parameters. Thus, the model was proposed to be a tool to distinguish which of the pulsars detected in gamma-rays could be good candidates to appear in X-rays. A list of pulsars appearing in the Second Fermi-LAT Pulsar Catalog (Abdo et al. 2013, 2PC hereafter) for which the theoretically expected X-ray flux is larger than $10^{-13} \mathrm{erg} \mathrm{cm}^{-2} \mathrm{~s}^{-1}$ at $10 \mathrm{keV}$, was provided in Torres (2018).

Enlarging the non-thermal X-ray pulsar population is clearly an important task: out of more than 200 gamma-ray pulsars known in the Galaxy, only less than $10 \%$ have been detected in X-rays (see e.g., Kuiper \& Hermsen 2015). Enlarging the sample of X-ray pulsars is crucial for understanding their global properties, and characterizing how these compare with those of other pulsar subsamples.

In this Letter, we take three pulsars out of the list in Torres (2018) and analyze the existing publicly available X-ray data for them. We find that pulsed radiation is indeed found for all of these pulsars, and provide an analysis of their spectral properties, comparing them with model predictions taking into account (or not) the now-determined X-ray data.

\section{Observation and Data Analysis}

We analyze archival XMM-Newton and Chandra data. The Chandra data were reduced using CIAO version 4.7 and CALDB version 4.7.7. We reprocessed the Chandra data to level $=2$ and removed periods of high background or flaring appearing in the observations. XMM-Newton data were reduced with the XMM-Newton Science Analysis System (SAS, version 16.1.0). Standard pipeline tasks (epproc for PN and emproc for MOS data) were used to process the raw observation data files (ODFs). XMM-Newton data were also filtered to avoid the periods of hard X-ray background flares. All of the X-ray spectra were rebinned to have at least 25 counts for each channel. Spectral analysis was carried out with XSPEC version 12.8.2.

From the spectral modeling of each pulsar we constructed the corresponding phase-averaged SED. These SEDs are produced by Xspec using the corresponding unabsorbed spectral models (i.e., the corresponding fitted pulsar's power-law models in Table 1) and the real exposure times and background.

For timing analysis, all times of arrivals of X-ray photons were barycenter-corrected using the position of the pulsars and the latest JPL DE405 Earth ephemeris. The pulsations were searched for via an $Z_{n}^{2}$-test procedure around the corresponding periods expected from the Fermi-LAT gamma-ray ephemeris or from previous publications, with Fourier resolution (Buccheri et al. 1983), and with the number of harmonics $n$ varied from 1 to 5 . We start from $Z_{1}^{2}$-test and increase $n$ until a significant signal is detected.

As we shall see below, the face-value periods that we find in X-rays-considering the uncertainty coming only from the Fourier resolution-are close, but not exactly at the gamma-ray pulsation period found earlier with Fermi-LAT. In addition to intrinsic phenomena in the pulsars, and the timing noise being 
Table 1

Spectral Fits to the X-Ray Pulsars

\begin{tabular}{lllcll}
\hline \hline Source Name & $\begin{array}{l}N_{H} \\
\left(10^{22} \mathrm{~cm}^{-2}\right)\end{array}$ & $\begin{array}{l}k T \\
(\mathrm{keV})\end{array}$ & Spectral Index $\Gamma$ & \multicolumn{2}{l}{$\begin{array}{l}\text { Unabsorbed Flux in 0.2-10 keV } \\
\left(10^{-13} \mathrm{erg} \mathrm{cm}^{-2} \mathrm{~s}^{-1}\right)\end{array}$} \\
\hline $\mathrm{J} 1747-2958$ & $2.58_{-0.24}^{+0.25}$ & $\cdots$ & $1.25 \pm 0.12$ & $23.46_{-1.04}^{+1.24}$ & $65.08 / 56$ \\
\hline $\mathrm{J} 2021+3651$ & 0.69 (fixed) & $0.15 \pm 0.01$ & $1.72 \pm 0.30$ & $0.78_{-0.09}^{+0.12}$ & $38.34 / 36$ \\
\hline $\mathrm{J} 1826-1256$ & $2.28_{-0.42}^{+0.49}$ & $\ldots$ & $1.31_{-0.20}^{+0.22}$ & $3.32_{-0.31}^{+0.47}$ & $17.29 / 16$ \\
& $0.79_{-0.26}^{+0.31}$ & $1.54_{-0.12}^{+0.13}$ & $\cdots$ & $2.01 \pm 0.12$ & $17.46 / 16$ \\
\hline
\end{tabular}

very large in at least two of the sources of our sample, we may consider other possible uncertainties that may lead to such differences. Among them, we consider the particular instrument timing resolution and accuracy, possible fluctuations of observed counts, and also the Fourier resolution.

To consider an uncertainty according to the corresponding timing resolution and accuracy, the arrival time of each event was uniformly sampled within its corresponding uncertainty to produce a simulated event list, which was later analyzed via the $Z_{n}^{2}$-test. This procedure was repeated 10,000 times for each source, leading to the estimation of a related period uncertainty. Similarly, to estimate the period uncertainty resulting from fluctuations of observed counts, we sampled within the phase bins of the corresponding folded lightcurves, assuming a Poisson distribution. Then the phases of sampled events are converted into arrival times in the observation to produce a simulated event list, which was again later analyzed via the $Z_{n}^{2}$-test. This process was also repeated 10,000 times for each source, leading to the estimation of a related period uncertainty. The errors in the periods were estimated as the sum in quadrature of the simulation obtained uncertainties and the Fourier resolution, with the former dominating the period error. In the folded pulse profile, an arbitrary reference epoch $T_{0}$ was set to the start of respective observations. The pulse profile was modeled by a sinusoidal function with $\mathrm{n}$ harmonics, while $\mathrm{n}$ was decided by the $Z_{n}^{2}$-test. The pulse fraction is defined as the ratio between the semi-amplitude of fundamental sinusoid and the average count rate. All of the uncertainties quoted in the spectral analysis are at $1 \sigma$ confidence level. Considering these uncertainties we find that the Fermi-LAT periods found earlier and the X-ray periods that we detect are compatible in all three cases studied.

\subsection{PSR J1747-2958: Detection of the Pulsar in the "Mouse" Nebula}

$\mathrm{J} 1747-2958$ is a $98.8 \mathrm{~ms}$ young pulsar, detected in radio and gamma-rays (see, e.g., Camilo et al. 2002; 2PC) associated with the axisymmetric nebula G359.23-0.82 (referred to as the Mouse). The Mouse nebula was discovered and studied in detail with Chandra (Gaensler et al. 2004; Klingler et al. 2018). In Gaensler et al. (2004), the head region of the Mouse nebula was decomposed into two Gaussian components. The first Gaussian was identified as a point-like source possibly associated with the pulsar itself, albeit no X-ray pulsations were earlier reported.

Chandra/High Resolution Camera (HRC) observed PSR J1747-2958 with $58 \mathrm{ks}$ exposure on 2008 February 7 (obsID 9106), providing sufficient timing accuracy ( $\sim 11.1 \mathrm{~ms}$, which is the median of time differences between observed events). ${ }^{7}$

\footnotetext{
7 http://cxc.harvard.edu/cal/Hrc/timing_200304.html
}

We considered this data set and searched for pulsations via the $Z_{n}^{2}$-test procedure. Photons were extracted with a radius of 1 arcsec using the position of the first Gaussian reported in Gaensler et al. (2004), which was proposed to be the pulsar magnetospheric emission (Gaensler et al. 2004). We found a peak at $P=0.09878(10) \mathrm{s}\left(90 \%\right.$ uncertainty) with a $Z_{2}^{2}$ statistic of 39.33 (Figure 1), which corresponds to a significance $\sim 5.3 \sigma$ after trials correction. The trials are the different values of $n$ (1 and 2) considered in $Z_{n}^{2}$-test. The latest Fermi-LAT gamma-ray ephemeris ${ }^{8}$ covers a range from 2008 August 13 to 2013 October 7. We extrapolated the gamma-ray ephemeris to the epoch of the X-ray observation. The expected pulse period is $P=0.098823858(4)$ s, which is compatible within the uncertainty of the period that we detected in X-rays. Additionally, we note that the timing noise is not considered in the pulse period prediction from gamma-ray ephemeris. Based on current LAT Gamma-ray Pulsar Timing Models, the timing noise of PSR J1747-2958 may reach values as large as $\sim 2 \times 10^{-5} \mathrm{~s}$ during the X-ray observation, leading to additional uncertainties in the predicted period. The HRC data were folded at the detected period and the pulse profile is also shown in Figure 1, yielding a pulse fraction of $20.9 \% \pm 4.7 \%$. Klingler et al. (2018) carried out a search of X-ray pulsations in the same Chandra/HRC data, but reported no detection. This is possibly due to different extraction regions, and/or a different number of events considered in the timing analysis. However, we note that the pulse fraction derived in our analysis is consistent with the upper limit calculated by Klingler et al. (2018; 34\%).

We carried out the spectral analysis of PSR J1747-2958 using archival Chandra observations (obsIDs 14519, 14520, 14521, 14522). The pulsar spectrum was extracted from an elliptical region centered on the first Gaussian component reported in Gaensler et al. (2004), having a major axis of 1.1 arcsec, an ellipticity of 0.25 , and a position angle of $118^{\circ}$ (see Figure 2, top-left panel). To exclude the contamination of the nebula, the background spectrum was extracted from an elliptical region centered on the second Gaussian component with a major axis of 2.4 arcsec, an ellipticity of 0.12 , and a position angle of $91^{\circ}$, excluding the region used for the pulsar spectrum extraction. To increase the statistics, spectra from different observations are combined using the task combine_spectra. We have tested that a simultaneous fitting to the spectra from different observations leads to consistent results. The combined spectra of PSR J1747-2958 could be well fitted with an absorbed power law (see Figure 3, reduced $\chi^{2}=1.16$, D.O.F $=56$ ). The fitted $N_{H}$ and flux level (Table 1) are consistent with the value reported in Gaensler et al. (2004). An absorbed blackbody could not lead to

\footnotetext{
8 LAT gamma-ray pulsar timing models, https://confluence.slac.stanford. edu/display/GLAMCOG/LAT+Gamma-ray+Pulsar+Timing+Models.
} 

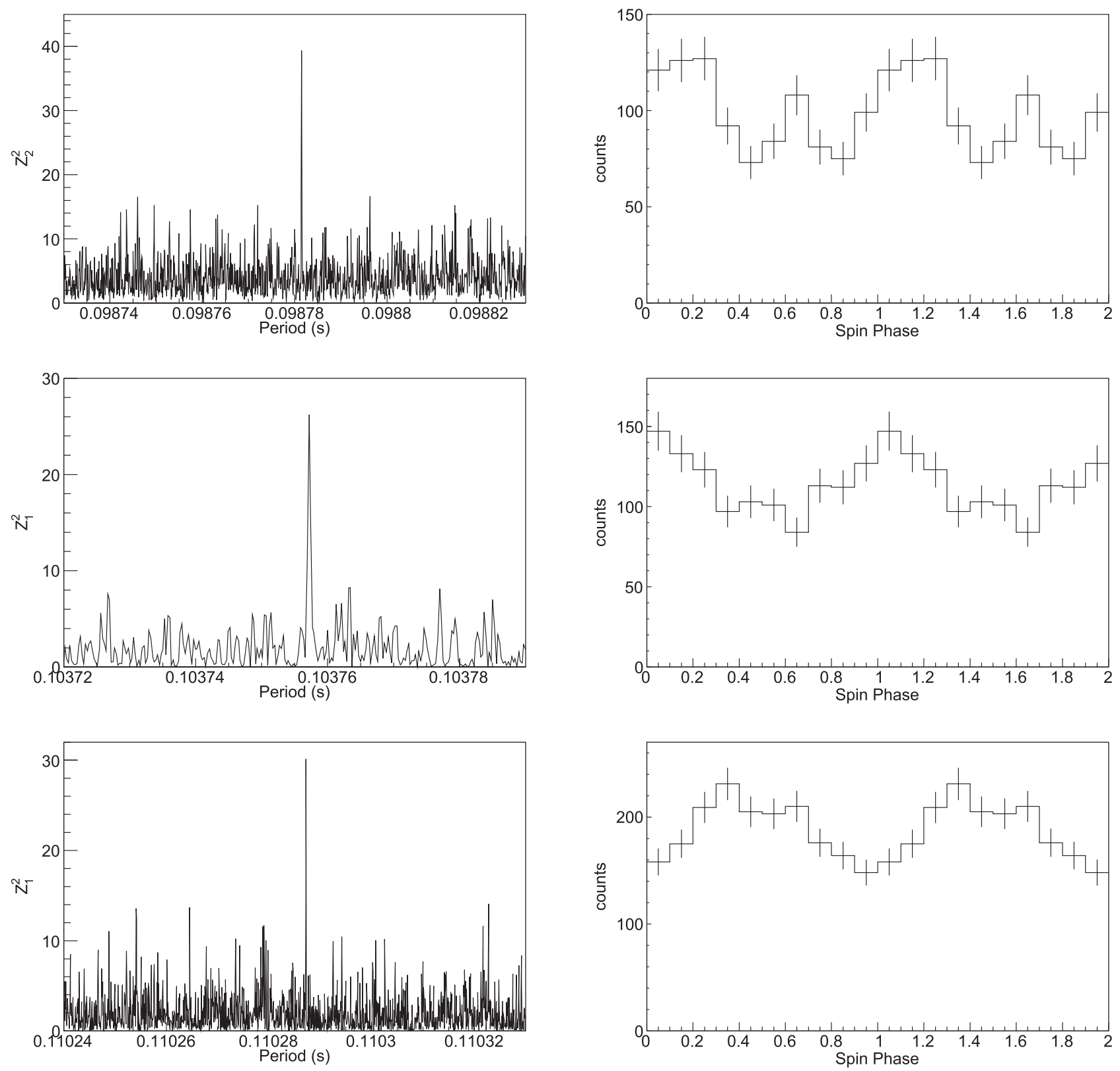

Figure 1. $Z_{n}^{2}$ periodogram (left panels) and pulse profile folded using the detected period (right panels). From top to bottom are PSR J1747-2958, PSR J2021+3651, and PSR J1826-1256). For the specific data used in each case, see the text.

an acceptable fit (reduced $\chi^{2}=1.7$, D.O.F $=56$ ). We also tested an absorbed power law plus blackbody model. However, the blackbody component is not significantly required according to the F-test (significance below $3 \sigma$ ). Thus, we conclude that the spectrum that we observe from PSR J1747-2958 is non-thermal.

\subsection{J2021+3651: Confirmed Detection of the Pulsar in the "Dragonfly" Nebula}

PSR J2021+3651 is a 103.7 ms radio and gamma-ray pulsar (Roberts et al. 2002; Abdo et al. 2009) associated with nebula G75.2+0.1 (referred to as the Dragonfly nebula; Hessels et al. 2004; Van Etten et al. 2008). In this case, hints of X-ray pulsations have earlier been reported using Chandra at a significance of $3.7 \sigma$ (Hessels et al. 2004). Here, we re-analyzed this Chandra observation.
Chandra observed PSR J2021+3651 on 2003 February 12 with ACIS-S operating in continuous clocking mode (obsID 3902 ). It provides $20 \mathrm{ks}$ exposure with sufficient timing resolution $(2.85 \mathrm{~ms})$. Adopting the X-ray position from Hessels et al. (2004), we extracted photons with a radius of 5 pixels. Because of the short exposure and low counts, we analyzed all of the events in $0.3-10 \mathrm{keV}$ via a similar $Z_{n}^{2}$-test procedure as described before (Buccheri et al. 1983). A peak at $P=0.10375$ (7) s (90\% uncertainty) is significantly detected with a $Z_{1}^{2}$ value of 26.21, corresponding to a significance $\sim 4.8 \sigma$. The radio predicted spin period is $0.10372423 \mathrm{~s}$ (Hessels et al. 2004), which is consistent with the X-ray detected period. The folded pulse profile is shown in Figure 1, leading to a pulse fraction of $19.86 \% \pm 4.27 \%$. The pulse profile is different from that reported in Hessels et al. (2004), which was produced using the radio expected period and $\mathrm{X}$-ray photons in $0.5-3 \mathrm{keV}$ from a extraction radius of 3 pixels. 

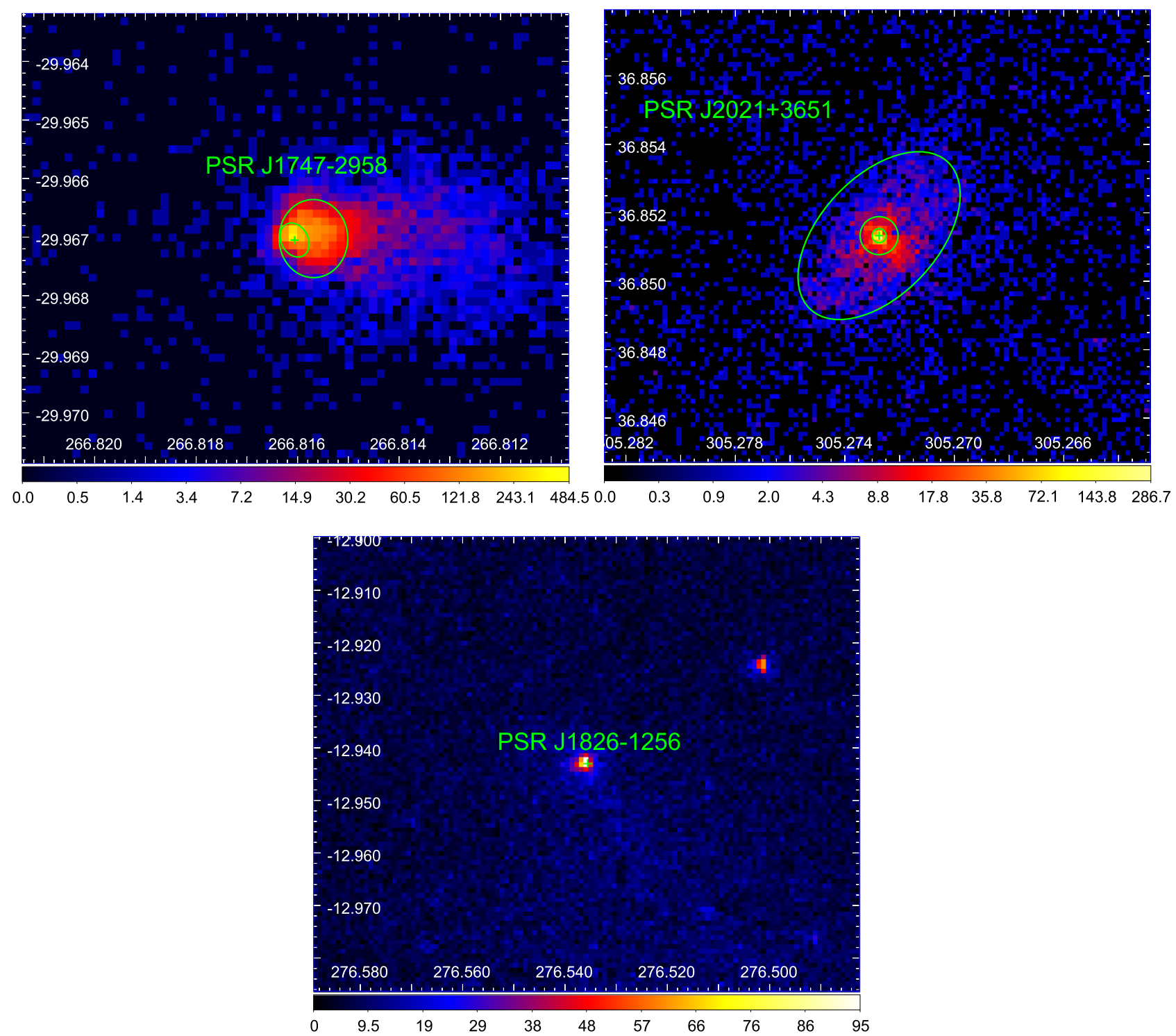

Figure 2. Log-scaled counts maps of the region around J1747-2958 (top left, Chandra/ACIS-I image in 0.3-10 keV, obsID 14519), J2021+3651 (top right, Chandra/ACIS-S image in 0.3-10 keV, obsID 7603), J1826-1256 (bottom, XMM-Newton/MOS1 and MOS2 combined image in 0.2-10 keV, obsID 0744420101). The positions of the pulsar are shown with green crosses. The regions used to extract the pulsar and nebula contributions in J1747-2958 and J2021+3651 are shown with ellipses and circles, respectively (see Sections 2.1 and 2.2 for details). The $X$ - and $Y$-axis are R.A. and decl. referenced at J2000.

We used the archival Chandra observations (obsIDs 3901, 7603 , 8502) for the spectral analysis of PSR J2021+3651, adopting similar methods to those described in Kirichenko et al. (2015) and Van Etten et al. (2008). The pulsar spectrum was extracted from all three of the observations using a source region of radius 1.5 pixels $(0.74$ arcsec). The nebula spectrum was extracted from an elliptical region with semi-axes of 6.2 and 10.6 arcsec and a position angle of $137^{\circ}$. A circle around the pulsar with a radius of 2 arcsec was excluded from this region (see Figure 2, top-right panel). Corresponding spectra are combined from the different observations to increase statistics, as similarly done for PSR J1747-2958. A simultaneous fitting to the spectra from different observations leads to consistent results. The spectrum of the nebula was fitted with an absorbed power law, leading to $N_{H}=(6.9 \pm 0.5) \times 10^{21} \mathrm{~cm}^{-2}$, photon index $\Gamma=$ $1.45 \pm 0.06$, and a flux level in the $0.2-10 \mathrm{keV}$ band of $7.47_{-0.18}^{+0.17} \times 10^{-13} \mathrm{erg} \mathrm{cm}^{-2} \mathrm{~s}^{-1}$, values that are consistent with previously published results (Hessels et al. 2004;
Van Etten et al. 2008; Kirichenko et al. 2015). As suggested by previous studies, the pulsar was modeled with an absorbed sum of the power law and blackbody components. The absorption column density was fixed at the nebula fitted value. To model the nebula contribution to the pulsar spectra, we added another power-law component to the pulsar spectral model, with a photon index fixed at the nebula value. Its flux level was fixed at the 5\% of total nebula flux, as suggested by Kirichenko et al. (2015) and Van Etten et al. (2008). Our fitting results for the pulsar are consistent with those of Kirichenko et al. (2015) and Van Etten et al. (2008; see Table 1).

\subsection{Detection of PSR J1826-1256}

PSR J1826-1256 is a 110.2 ms radio quiet gamma-ray pulsar discovered by Fermi-LAT (Ray et al. 2011). Its X-ray counterpart has been identified with the Advanced Satellite for Cosmology and Astrophysics (ASCA) and Chandra 

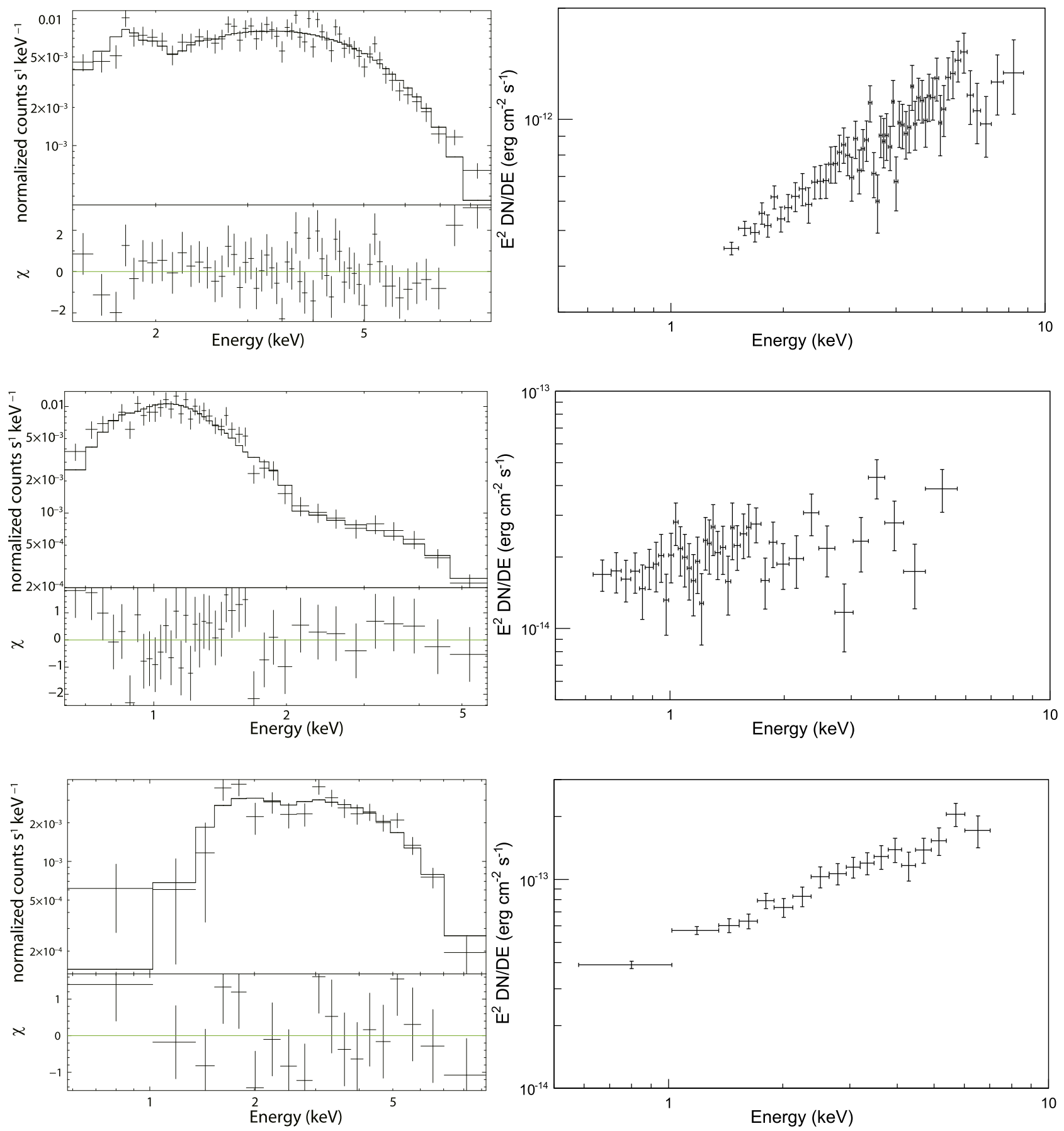

Figure 3. The left panels show the spectrum of J1747-2958 (top), J2021+3651 (middle), both with Chandra/ACIS-S data; and J1826-1256 (bottom), with combined XMM-Newton/MOS1 and MOS2. The best-fitted models and post-fit residuals are also shown in each case. The right panels show the corresponding pulsar X-ray spectral energy distributions (only the non-thermal spectral component is shown for J2021+3651).

(Roberts et al. 2001; Ray et al. 2011) but no X-ray pulsations were reported.

XMM-Newton observed PSR J1826-1256 with $140 \mathrm{ks}$ exposure, from 2014 October 11 to 13 (Figure 2, bottom panel). During this observation, PN was operating in small window mode, providing sufficient time resolution $(5.7 \mathrm{~ms})$ to search for X-ray pulsations. Using the X-ray position from Ray et al. (2011), we searched for pulsations of J1826-1256 via the $Z_{n}^{2}$-test procedure. We extract photons from PN data using a radius of 10 arcsec in $0.3-10 \mathrm{keV}$. We found a peak at $P=0.11028(5)$ s $\left(90 \%\right.$ uncertainty; see Figure 1). The $Z_{1}^{2}$ statistic of this peak is 30.12 , which corresponds to a significance $\sim 5.1 \sigma$. We folded the extracted PN data at the detected period and the pulse profile is also shown in Figure 1, yielding a pulse fraction of $18.06 \% \pm 3.26 \%$. The latest FermiLAT gamma-ray ephemeris covers the period from 2008 August 8 to 2013 October 18. We extrapolated the gamma-ray ephemeris to the epoch of the X-ray observation. The extrapolated pulse period is $P=0.11024444(2) \mathrm{s}$, which is within the uncertainty of the period that we detected. When extrapolating the gamma-ray ephemeris, timing noise has not been considered. Based on current LAT Gamma-Ray pulsar 

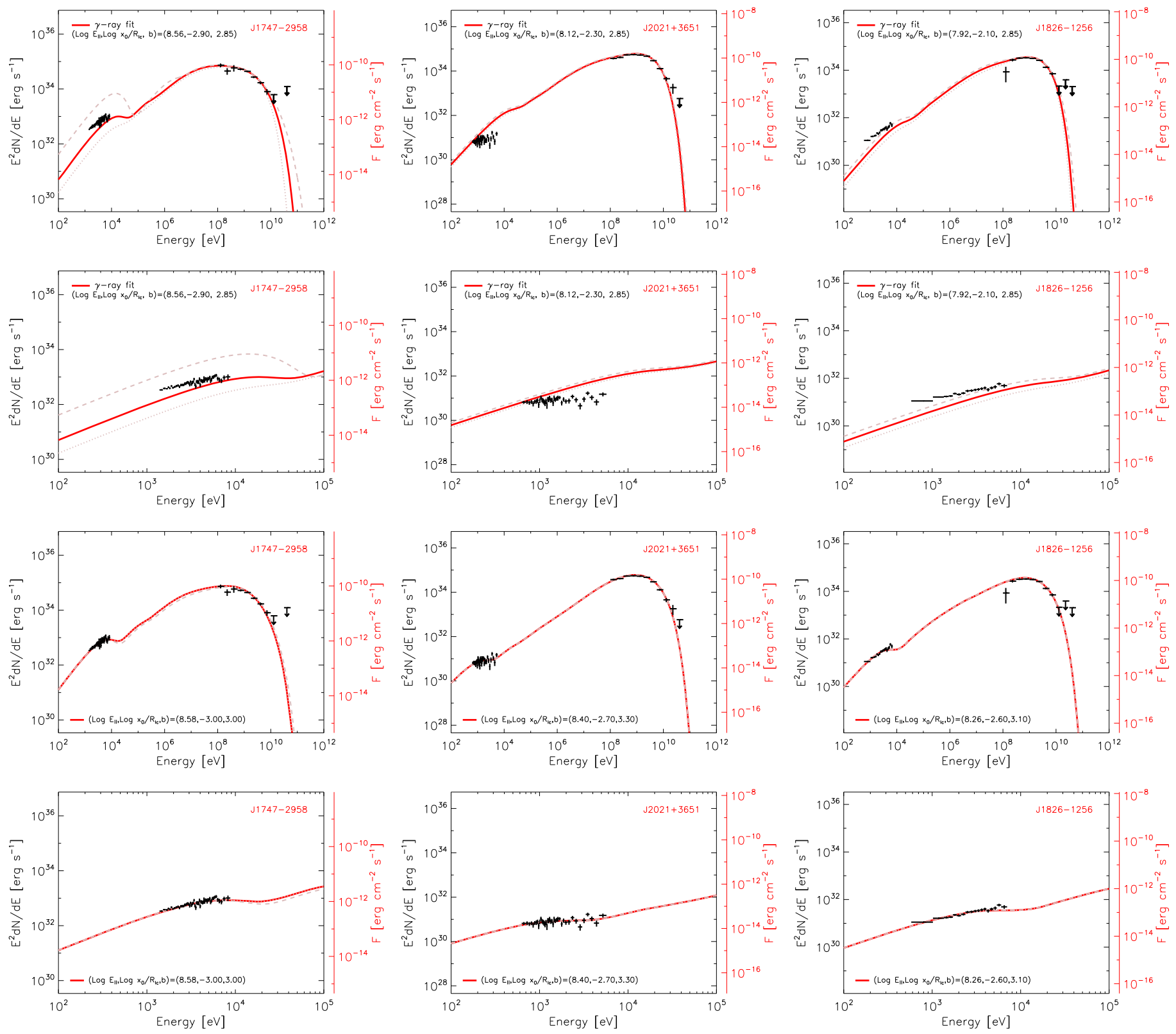

Figure 4. The first and second rows from the top show the unabsorbed non-thermal X-ray spectra as determined in this Letter plotted against the model with fixed magnetic gradient $(b=2.85$ see the text for an explanation) used to select these pulsars as possibly detectable. In these rows only the gamma-ray data are fitted, not the X-ray data. The third and fourth rows show the model fittings considering both the X-ray and gamma-ray data. The red line is the fitted model in all cases, while the dotted lines (when visible) indicate $1 \sigma$ uncertainty in the fitting parameters. The second and fourth rows show a zoom of the X-ray band for the corresponding firstand third-row panels. The non-thermal X-ray SEDs are taken from the right panel of Figure 3.

timing models, the timing noise of PSR J1826-1256 may reach values as large as $\sim 4 \times 10^{-4}$ s during the X-ray observation, and leads to additional uncertainties in the predicted period.

PSR J1826-1256 is detected as a point-like source with XMMNewton. The MOS1 and MOS2 combined image of J1826-1256 in $0.2-10 \mathrm{keV}$ is shown in Figure 2. The X-ray spectra for J1826-1256 were extracted separately from MOS1 and MOS2 data, and then combined using the task epicspeccombine to increase statistics. We also have tested that a simultaneous fitting to the spectra from MOS1 and MOS2 leads to consistent results. The background was subtracted, extracted from a source-free region near PSR J1826-1256 (see Figure 2). The pulsar spectrum could be well fitted with an absorbed power law (see Figure 3 and Table 1). An absorbed blackbody could also lead to acceptable fit. We could not distinguish both models directly with the current statistics. However, as we note below, the pulsar spectrum is consistent with the non-thermal model prediction.

\section{Discussion}

Figure 4 shows the derived non-thermal SEDs for all three pulsars in comparison with models. The first two rows show the initial prediction. The model is described in detail in Torres (2018) and references therein. It encompasses two essential ingredients. On the one hand, it contains a dynamical, time/ position-dependent description of particle trajectories in an accelerating region in the outer part of the magnetosphere, where particles are subject to radiative losses. On the other hand, it features a computation of the spectrum emitted at each position, while particles traverse the accelerating region. For 
both losses and radiation, the full synchro-curvature process is considered (see Viganò et al. 2015b for a description). Just three physical parameters (which we mention below), together with the timing properties of the pulsar (i.e., the measured period and period derivative), define the shape of spectrum.

The red line in each panel of Figure 4 is the fitted model in all cases. The panels in the second row show a zoom of the region in the X-ray band for each of the corresponding first-row panels. In all three panels of the first row the model is obtained as a fit to the gamma-ray data only. Such data are lying at energies that are six orders of magnitude larger than the spectrum that we have now determined. These red lines represent the theoretical prediction that we used in order to consider that these three pulsars were actually detectable in $\mathrm{X}$-rays. In deriving such predictions, the model used only two physical parameters: the accelerating electric field, $E_{\|}$, and the contrasting $\left(x_{0} / R_{l}\right)^{-1}$. The latter is a description of the uniformity of the particle distribution along the accelerating region. The third physical parameter of the model, the magnetic gradient, $b$, representing a measure of how fast the magnetic field declines along the particle trajectory, was kept fixed. The value of $b$ in these initial fits was assumed to be 2.85 , and not fitted against. This value is the average found for the pulsars detected in non-thermal X-rays (above $20 \mathrm{keV}$ ) and gammarays studied by Torres (2018). As it was discussed earlier (Viganò et al. 2015a; Torres 2018), having only gamma-ray above $100 \mathrm{MeV}$ makes for a difficult distinction among different values of $b$. The values of these parameters are shown in Figure 4. The agreement between the X-ray predictions of these models and the determined spectral data is impressive, which confirms that the model works well to select which pulsars among those detected in gamma-rays are detectable in X-rays. This fact can then be used to further enlarge, as we do here, the sample of non-thermal pulsars detected in the X-rays, which is still small (see e.g., Kuiper \& Hermsen 2015).

The third row of Figure 4 shows the model fits obtained considering also the X-ray data, whereas the fourth row zooms into the X-ray region. These fits have a free value of magnetic gradient, and were obtained spanning uniformly on $E_{\|}, b$, and $x_{0}$. These three physical parameters provide a correct description of the whole multiwavelength data set. All three fits can cope well with both sets of data in such different energy regimes, confirming that the model is generally applicable. In all three cases, whereas the values of the accelerating electric field $E_{||}$and the contrast $\left(x_{0} / R_{l}\right)^{-1}$ are roughly unchanged from the gamma-ray-only fits, the chosen magnetic gradients are larger. The value for $\mathbf{J} 2021+3651(b=3.30)$ is in fact the largest of the magnetic gradients found till now for all X-ray and gamma-ray detected pulsars (see Torres 2018), with all three being comparable to Vela $(b=3.25)$ or PSR J2022 $+3842(b=3.10)$. Larger values of $b$ make the spectrum softer at X-ray energies (predicting larger fluxes at soft X-ray energies; see Supplementary Figure 2 of Torres 2018). If more normal pulsars would be better described by values of magnetic gradients larger than 2.85 , the current average value, the number of possible X-ray detectable pulsars will increase. This is something that future studies using new samples of gammaray pulsars (e.g., the forthcoming Third Fermi Pulsar Catalog) should take into account.

We acknowledge the support from The National Key Research and Development Program of China (2016YFA0400800), the Spanish grants AYA2015-71042-P, SGR2017-1383, AYA201792402-EXP, iLink 2017-1238, and the National Natural Science Foundation of China via NSFC-11473027, NSFC-11503078, NSFC-11673013, NSFC-11733009, NSFC-U1838201, XTP project XDA 04060604 and the Strategic Priority Research Program "The Emergence of Cosmological Structures" of the Chinese Academy of Sciences, grant No. XDB09000000. Jian Li acknowledges the support from the Alexander von Humboldt Foundation. Alessandro Papitto acknowledges the agreements ASI-INAF I/037/12/0 and ASI-INAF 2017-14-H.O, and the Marie Skłodowska-Curie grant agreement 660657-TMSPH2020-MSCA-IF-2014. We acknowledge the support of the PHAROS COST Action (CA16214). We acknowledge discussions with Dr. Long $\mathrm{Ji}$, and help from the XMM-Newton \& Chandra Helpdesk staff. Work at NRL is supported by NASA.

\section{ORCID iDs}

Jian Li (iD https:// orcid.org/0000-0003-1720-9727

Diego F. Torres (ib https://orcid.org/0000-0002-1522-9065

Alessandro Papitto (1) https://orcid.org/0000-0001-6289-7413

Matthew Kerr (i) https://orcid.org/0000-0002-0893-4073

Nanda Rea (iD https://orcid.org/0000-0003-2177-6388

\section{References}

Abdo, A. A., Ackermann, M., Ajello, M., et al. 2009, ApJ, 700, 1059 Abdo, A. A., Ajello, M., Allafort, A., et al. 2013, ApJS, 208, 17 Buccheri, R., Bennett, K., Bignami, G. F., et al. 1983, A\&A, 128, 245 Camilo, F., Manchester, R. N., Gaensler, B. M., \& Lorimer, D. R. 2002, ApJ, 579,25

Gaensler, B. M., van der Swaluw, E., Camilo, F., et al. 2004, ApJ, 616, 383 Hessels, J. W. T., Roberts, M. S. E., Ransom, S. M., et al. 2004, ApJ, 612, 389 Kirichenko, A., Danilenko, A., Shternin, P., et al. 2015, ApJ, 802, 17 Klingler, N., Kargaltsev, O., Pavlov, G. G., et al. 2018, ApJ, 861, 5 Kuiper, L., \& Hermsen, W. 2015, MNRAS, 449, 3827

Ray, P. S., Kerr, M., Parent, D., et al. 2011, ApJS, 194, 17 Roberts, M. S. E., Hessels, J. W. T., Ransom, S. M., et al. 2002, ApJ, 577, 19 Roberts, M. S. E., Romani, R. W., \& Kawai, N. 2001, ApJS, 133, 451 Torres, D. F. 2018, NatAs, 2, 247

Van Etten, A., Romani, R. W., \& Ng, C.-Y. 2008, ApJ, 680, 1417 Viganò, D., Torres, D. F., \& Martin, J. 2015a, MNRAS, 453, 2599

Viganò, D., Torres, D. F., Pessah, M., \& Hirotani, K. 2015b, MNRAS, 447, 1164 